\title{
Veille d'image sur Internet : enjeux, méthodes, limites
}

Eric Boutin, Pei Liu et Lysiane Buisson

\section{(2) OpenEdition}

1 Journals

Édition électronique

URL : http://journals.openedition.org/communicationorganisation/611

DOI : 10.4000/communicationorganisation.611

ISSN : $1775-3546$

Éditeur

Presses universitaires de Bordeaux

Édition imprimée

Date de publication : 1 décembre 2008

Pagination : 98-114

ISBN : 978-2-86781-582-9

ISSN : $1168-5549$

Référence électronique

Eric Boutin, Pei Liu et Lysiane Buisson, «Veille d'image sur Internet : enjeux, méthodes, limites », Communication et organisation [En ligne], 34 | 2008, mis en ligne le 01 décembre 2011, consulté le 01 mai 2019. URL : http://journals.openedition.org/communicationorganisation/611 ; DOI : 10.4000/ communicationorganisation.611 


\title{
Dossier : Retour sur les images d'organisations
}

\section{Résumé :}

La « veille d'image » consiste, pour une organisation, à identifier le plus en amont possible des signaux faibles négatifs de type rumeur ou attaque informationnelle. Notre travail est centré sur la «veille d'image sur Internet ». Cette notion, qui a fait l'objet de peu de travaux académiques, est surtout abordée par des consultants. Certains d'entre eux forcent le trait du risque encouru pour vendre leurs services. L'objectif de ce papier est de proposer un discours plus nuancé sur la portée « virale » de l'Internet. Cette étude repose sur deux études de cas qui montrent que les risques d'attaques contre l'image d'une organisation ne seraient pas aussi grands qu'annoncés par les vendeurs de logiciels de surveillance. Ce travail met en évidence l'existence d'une logique d'autorégulation qui agit comme une «main invisible » pour contrebalancer les critiques exprimées.

\section{Mots-clefs}

Veille d'image, web 2.0, blogosphère, rumeur, analyse webométrique, sagesse des foules

\begin{abstract}
"Image awareness" consists to identify as soon as possible negative weak signals like rumors or informational attacks. Our work focuses on the analysis of the image of an organization through the Internet. This concept is primarily addressed by consultants. Some of them enhance the risks to sell their services. This paper proposes a more nuanced discourse on the scope "viral" of the Internet. It is based on two case studies which show that the risk of attacks against the image of an organization would not be as announced by major vendors of monitoring softwares. This work reveals the existence of such autoregulated mecanisms like an "invisible hand" that offset the expressed criticisms.
\end{abstract}

\section{Key-Words}

Reputation management, web 2.0, blogosphere, rumor analysis, webometric analysis, wisdom of crowds

Eric Boutin est Maître de Conférences en Sciences de l'Information et de la Communication à l'Université du Sud Toulon Var. Il est chercheur au sein du laboratoire I3M dans cette Université.

Pei Liu est doctorante en Sciences de l'Information et de la Communication à l'Université du Sud Toulon Var. Elle occupe un poste d'ATER et effectue ses recherches au sein du laboratoire I3M dans cette Université.

Lysiane Buisson est doctorante en Sciences de l'Information et de la Communication. Elle occupe un poste de PRAG à l'Université du Sud Toulon Var et effectue ses recherches au sein du laboratoire I3M dans cette Université. 
Veille d'image sur Internet...

\title{
Veille d'image sur Internet : enjeux, méthodes, limites
}

Eric Boutin, Pei Liu, Lysiane Buisson

\author{
boutin@univ-tln.fr \\ liu@univ-tln.fr \\ buisson@univ-tln.fr
}

De nombreux travaux étudient la relation entre l'image de marque d'une organisation et ses indicateurs de performance économique et sociale: une bonne image permettrait par exemple d'attirer ou de conserver des collaborateurs talentueux, de conquérir ou de fidéliser des consommateurs, de séduire ou de rassurer des investisseurs. On comprend alors l'importance stratégique pour l'organisation de construire et de préserver son capital image. Appréhender l'image de marque dans une perspective dynamique est d'autant plus important pour l'organisation que l'image a souvent un fort degré de volatilité, de versatilité, d'instabilité. Elle met généralement du temps à se construire mais peut a contrario se dégrader très rapidement ${ }^{1}$. Cette instabilité est inhérente à la nature même de l'image de marque. Nous la définirons ici comme l'image symbolique perçue de l'organisation par le public. Elle résulte d'une série de messages perçus comme exprimant l'identité de l'organisation et détermine l'attitude du public vis-à-vis de l'organisation. Nous considérons que cette représentation mentale est subjective, sélective et simplificatrice et qu'elle évolue selon une logique qui tient de la rationalité limitée. Selon Barnett (2006), l'image perçue (Christie - 2002) se construit davantage à partir de considérations émotionnelles et subjectives que rationnelles. C'est ce qui fait dire à John Kay (2004) que l'image de l'organisation est le principal moyen par lequel une économie de marché traite l'ignorance des consommateurs.

Cette sensibilité de l'image justifie l'intérêt d'une surveillance continuelle de l'environnement de l'organisation pour détecter des éléments qui pourraient la dégrader. Nous parlons de «veille d'image » pour caractériser ce processus de vigilance permanente qui se traduit concrètement par une démarche de collecte, de traitement et d'analyse humaine et automatique d'informations. Par veille d'image nous n'entendons donc pas la simple construction d'un baromètre

\footnotetext{
${ }^{1}$ Le spécialiste de l'image voudra bien excuser ce raccourci rapide qui a pour objet de mettre en avant la pertinence de notre approche du sujet.
} 


\section{Dossier : Retour sur les images d'organisations}

permettant de mesurer l'évolution de l'image de l'organisation au fil du temps. L'objectif est ici d'identifier le plus tôt possible des signaux faibles de risque d'image. Selon Olivier Mevel (2004), les signaux faibles sont des informations partielles et fragmentaires fournies par l'environnement. Si elles ne sont pas perçues et traitées à temps, l'organisation court le risque de voir son image se dégrader. L'enjeu est crucial mais la tâche est délicate puisqu'il s'agit de détecter les informations susceptibles d'affecter les éléments périphériques constitutifs de la représentation mentale qu'a le public de l'organisation et d'évaluer leur capacité à la déstabiliser. L'analyse est d'autant plus complexe en raison de la surcharge informationnelle induite par la diversité des acteurs potentiellement impliqués et de la multiplicité des supports possibles.

Notre travail est centré sur la « veille d'image sur Internet ». Le réseau est ici considéré comme une source d'information privilégiée qui s'exprime à travers une grande variété de supports (web, blogs, forums...) et une multiplicité d'auteurs (développement des Consumer Generated Media - CGM, développement du web 2.0). La singularité de cette source d'information tient d'une part au rapport temps-espace induit par l'immédiateté de la diffusion de l'information et d'autre part à la logique d'interactions qui préside à son fonctionnement (interactions par le lien hypertexte entre des acteurs d'un forum par exemple).

Il est sans doute temps de réajuster sur cette base la définition primitive que nous avions donnée de la veille d'image sur Internet. Il ne s'agit plus seulement d'identifier les signaux faibles. Il faut comprendre les ressorts de la diffusion d'une information sur Internet et agir au même titre que les internautes acteurs. On désignera donc par veille d'image sur Internet un processus visant d'une part l'identification de signaux faibles, émis sur Internet, susceptibles d'altérer l'image de l'organisation et d'autre part la mise en oeuvre d'actions correctives destinées à éviter que ces signaux faibles ne dégradent l'image de l'organisation étudiée.

La notion de veille d'image sur Internet a fait l'objet de peu de travaux académiques. Le sujet semble être accaparé par des consultants. La logique marchande conduit certains d'entre eux à forcer le trait du risque encouru pour vendre leurs services. On insiste, exemples à l'appui, sur l'effet dévastateur d'une communication virale non maitrisée. Le «bouche à oreille électronique » par lequel les internautes, par intérêt personnel, amusement ou simple curiosité, transmettent un message avec recommandation explicite ou implicite 
sur l'organisation à leurs réseaux de connaissances ou aux autres membres d'un espace d'échange, doit absolument être pris en considération par l'organisation dans une logique de marketing viral. On assiste en effet à la croissance exponentielle de l'exposition au message et à son influence directe sur l'image de marque de l'organisation (Jurvetson, Draper, 2000 ; Wilson, 2000). Cette logique commerciale conduit également à privilégier une approche technique en présentant des robots permettant de collecter automatiquement des informations sensibles pour l'entreprise. Notre objectif dans cette communication est d'évaluer la pertinence de ce discours dominant.

La veille sur Internet menée par le secteur marchand est-elle apte à rendre compte de l'image de l'organisation ? Où placer le curseur indiquant la nécessité de mettre en place des actions correctives sur l'échelle de dangerosité d'un signal faible ?

Pour répondre à ces questions, nous allons nous appuyer au-delà de considérations théoriques, sur un travail de terrain au contour bien délimité qui va consister à réaliser une veille sur Internet dans un contexte professionnel, celui des banques françaises, avec un focus particulier sur la « Caisse d'Epargne ».

Afin de ne pas répondre à ces questions de façon trop hâtive, nous avons choisi d'étudier deux supports d'information qui permettent une approche de l'image plus ou moins focalisée. L'image de l'entreprise est un objet d'étude polymorphe que l'on peut aborder selon plusieurs angles. On peut notamment s'intéresser à l'entreprise employeur, à l'entreprise titre, à l'entreprise citoyenne ou encore à l'entreprise productrice. Ces facettes sont bien évidemment indissociables et interdépendantes et centrer notre analyse a priori sur l'une de ces dimensions serait une simplification réductrice. Toutefois, certains supports d'information sur Internet sont des sources d'information qui ciblent de fait plus particulièrement l'une ou l'autre de ces dimensions. C'est le cas des sites de recommandation qui permettent de connaître l'opinion des clients. En interrogeant les blogs, on se trouve en revanche confronté à une information beaucoup plus dispersée.

Nous analyserons dans un premier temps la perception qu'ont les clients de la Caisse d'Epargne de leur banque à travers l'étude d'un site web de recommandation. Nous nous intéresserons ensuite aux mécanismes qui s'opèrent au sein de la blogosphère (ensemble des blogs). Dans les deux cas, nous effectuerons une analyse critique des méthodes d'analyse automatiques qui peuvent être mises en œuvre. 


\section{Dossier : Retour sur les images d'organisations}

\section{Veille d'image sur un site de recommandation}

Un site de recommandation est un site web dans lequel des consommateurs expriment publiquement des opinions (positives ou négatives) relatives à leur consommation de produits ou de services. Ces observations sont alors classées par rubriques et consultables par des internautes à la recherche d'informations destinées à étayer par exemple une décision d'achat future. Ces commentaires primitifs peuvent être enrichis par d'autres, générant sur certains sujets de véritables discussions contradictoires. Notre travail va porter sur le site de recommandation $\mathrm{Ciao}^{2}$.

Un sondage réalisé en 2006 par l'institut Ipsos $^{3}$ a souligné l'importance de la prise en compte de ce type de sites web dans la décision d'achat des internautes. Cette étude révèle en effet deux informations intéressantes. Les Français accordent deux fois plus de crédit à l'avis d'autres consommateurs sur le net qu'à une publicité. De plus, seuls $44 \%$ des internautes interrogés affirment ne pas avoir été influencés dans leurs décisions d'achat par les avis et commentaires d'autres clients ou particuliers qu'ils ont pu lire sur Internet. Ces deux chiffres semblent justifier l'intérêt que doit porter une organisation à l'identification de signaux faibles sur ce type de site.

Ces sites de recommandation fournissent une information qui peut être analysée à plusieurs niveaux. Tout d'abord les interventions dans un site de recommandation sont l'expression spontanée d'opinions sur un produit ou un service. Les internautes qui prennent connaissance de ces messages peuvent à leur tour se forger une opinion sur le bien ou service. Ces messages peuvent donc être analysés d'une part au niveau du signal émis par certains acteurs (des clients réguliers, des clients occasionnels, des salariés de la banque, des professionnels du secteur bancaire) et d'autre part au niveau du signal reçu par d'autres (les acheteurs potentiels) qui contribuera à forger, affiner ou modifier leur image perçue.

Dans cette partie, nous aborderons successivement la question de la difficile perception des signaux faibles, la mesure de leur niveau de dangerosité pour conduire à un profilage des acteurs intervenant sur un site de recommandation.

2 http://www.ciao.fr

3 Quel est le pouvoir économique des blogs en Europe institut Ipsos http://loiclemeur.com/IPSOSeuroblogs2006.pdf 
Veille d'image sur Internet...

\section{Percevoir les signaux faibles}

La question centrale de ce travail est celle de l'identification de phénomènes émergents qui peuvent à terme porter atteinte à l'image de l'entreprise. Toute la difficulté de leur détection tient au fait que les changements ne sont pas soudain mais progressifs, graduels et donc difficilement perceptibles. Cipollone (2006) expose le cas d'une pauvre grenouille pour faire comprendre le phénomène. $\mathrm{Si}$ on plonge une grenouille dans un seau d'eau très chaude, elle en sortira immédiatement. Si on plonge la même grenouille dans un seau d'eau à température ambiante et qu'on chauffe cette eau imperceptiblement, la pauvre grenouille ne se rendra pas compte du changement de la température et mourra ébouillantée. Ce mécanisme est un biais cognitif bien connu en recherche d'information. Heuer (1999) définit un biais cognitif comme une «erreur mentale causée par des stratégies de traitement simplifié de l'information». Dans le contexte présent, on s'intéresse à la dissonance cognitive (Festinger - 1957) associée à la prise en compte par l'individu d'une information qui ne renforce pas la connaissance qu'il a accumulée sur le sujet. Lorsqu'on fait une veille d'image, on aborde de nouveaux documents. Un contenu différent de la représentation que l'analyste s'est forgée va introduire un phénomène de dissonance cognitive. Une solution pour revenir à un état normal est de ne pas prendre en compte ces nouvelles informations. Ainsi, il sera très difficile pour un texte qui n'est pas confirmatoire des précédents d'être retenu comme pertinent. Il y a donc une forme d'aveuglement à la perception de signes émergents. Robson (2005) parle de verrou cognitif qu'il s'agit de faire sauter. La veille sur Internet suppose un état d'esprit ouvert aux possibles. La réalisation de ce travail par un consultant étranger à l'organisation et la supposée neutralité des outils d'analyse automatique qu'il utilise peut être un moyen de s'affranchir des idées préconçues.

Pour autant, doit-on accorder une confiance absolue aux méthodes couramment utilisées dans le secteur marchand de la veille sur Internet? Les logiques d'analyse textuelle automatique de corpus web volumineux sous-jacentes sont-elles aptes à répondre à cet objectif ambitieux ?

\section{Evaluer la dangerosité des signaux faibles}

A un premier niveau d'analyse il est tentant d'utiliser l'information brute contenue dans ces sites de recommandation pour mesurer l'image de l'entreprise auprès de ses clients. Tous les messages concernant une entreprise sont regroupés dans une rubrique identifiée. Il est alors aisé en appliquant des algorithmes d'analyse textuelle 


\section{Dossier : Retour sur les images d'organisations}

d'identifier les interventions négatives ou positives. Cette mesure automatique de la polarité d'un texte a fait l'objet de travaux en linguistique computationnelle. De façon générale et simplificatrice, l'orientation positive ou négative d'un texte est déterminée à partir de l'orientation positive ou négative du vocabulaire utilisé dans ce texte (Lacey - 2005). La polarité des mots d'un texte s'effectue en confrontant les mots du texte à un dictionnaire de polarité. Le General Inquier est, par exemple, un dictionnaire qui associe une mesure discrète de polarité à 4206 termes de la langue anglaise. Le Whissell's dictionary of affect in langage (Whissell, 1989) fournit une liste de 8242 termes dont la polarité est définie de façon continue. Pour la langue française, un dictionnaire a été élaboré par le département de psychologie de l'université Catholique de Louvain (Belgique). Ce dictionnaire comporte 5744 entrées ce qui correspond à 35459 termes non lemmatisés de la langue française. Il a été élaboré par au moins 30 juges qui ont qualifié le caractère déplaisant (1) ou plaisant (7) de chaque mot sur une échelle à 7 modalités. Chaque terme de ce dictionnaire est défini par une mesure continue de sa polarité comprise entre 15 (terme à polarité très négative) et 70 (terme à polarité très positive). Une fois que la polarité des termes d'une page est connue, l'objectif est d'agréger les résultats pour obtenir la polarité d'un ensemble de mots. Ce passage d'une analyse au niveau du mot à une analyse au niveau d'un texte oblige à maîtriser deux contraintes. La première concerne les règles linguistiques de la négation. La phrase «je ne recommande pas cette banque » a une polarité négative et la méthode doit permettre d'inverser la polarité d'un terme lorsque celui ci se trouve dans une tournure négative. La seconde contrainte vient du fait que certaines unités lexicales composées ont une polarité différente des polarités des termes qui la composent. L'expression «avoir mangé son pain blanc» a une polarité plutôt négative alors qu'aucun de ces termes n'a de polarité négative.

En mettant en œuvre ces techniques de calcul de polarité d'un texte, il est possible de calculer le pourcentage d'interventions favorables, défavorables aux produits ou services de l'entreprise. Nous nous sommes livrés à ce type d'analyse sur un corpus de 49 interventions concernant la Caisse d'Epargne publiées entre le 1er janvier 2007 et le 20 Avril 2008. Cette mesure de polarité dichotomique ne peut traduire à elle seule la richesse et la finesse de la qualification de l'image de l'organisation.

Les résultats montrent qu'environ $80 \%$ des messages sont hostiles à la Caisse d'Epargne. L'image projetée de la banque sur ce site est 
fortement négative mais il est nécessaire d'interpréter ces statistiques avec prudence.

Les prises de position négatives ont plus de chances de s'exprimer sur le web. Un client non satisfait aura un penchant plus naturel à verbaliser sa critique qu'un client satisfait à exprimer son contentement.

Une exposition à des textes majoritairement négatifs risque de laisser des traces négatives dans la perception de l'image de l'organisation dans l'esprit de l'internaute client potentiel de la banque. Il est à craindre qu'intervienne un biais cognitif appelé loi des petits nombres qui amplifier le phénomène. Cette loi, étudiée par Tversky et Kahneman (1974), Kahneman et $a$ l (1982) est une parodie de la loi des grands nombres connue en statistique. La loi des grands nombres repose sur un principe selon lequel des mesures conduites à partir d'échantillons représentatifs d'une population mère pourront ensuite être transposés à la population entière. Parler de «loi des petits nombre » correspond à l'idée selon laquelle les internautes ont du mal à apprécier la représentativité de l'information qu'ils exploitent et attribuent une confiance excessive à des conclusions issues de l'exploitation d'informations obtenues à partir d'échantillons non significatifs. L'internaute a une mauvaise évaluation de la représentativité d'une information et peut par exemple considérer la simple répétition d'une même information comme la confirmation de celle-ci.

La configuration du site de recommandation Ciao peut favoriser ce biais : les réponses pour chaque thème sont nombreuses, les réponses s'affichent par 10 ce qui renforce cette impression d'abondance, chaque intervention donne lieu à environ 5 commentaires exprimés par d'autres internautes. Lorsque l'internaute aborde l'interface de Ciao et qu'il navigue sur ce site, il peut avoir le sentiment d'appartenir à une communauté élargie d'acteurs représentatifs des clients de la banque.

Inférer l'intensité du signal reçu à partir d'une simple mesure de l'intensité du signal émis est abusif. Certains biais vont agir comme amplificateurs de signal, a contrario les visiteurs mettent sans doute en œuvre des filtres obéissant à une logique rationnelle (conscience de l'effet de miroir déformant du web par exemple) ou subjective (dissonance cognitive) face aux informations négatives disponibles sur le site. Il existe un déphasage entre l'intensité du signal négatif émis et sa dangerosité pour l'image perçue de l'entreprise. 


\section{Dossier : Retour sur les images d'organisations}

Une mesure quantitative du signal émis peut éventuellement être utilisée dans une logique diachronique ou être comparée avec les chiffres obtenus par les concurrents dans un même contexte d'analyse. Une augmentation des signaux négatifs dans le temps ou une analyse concurrentielle défavorable pourraient être interprétées comme un accroissement du danger potentiel pesant sur l'image de l'entreprise. Cependant, la détermination du degré de dangerosité signifiant, nécessitant la mise en place d'actions correctives, demeure une décision arbitraire.

Identifier les profils des intervenants sur un site de recommandation

Il est important pour l'organisation concernée de décrypter le jeu des acteurs qui interviennent et s'expriment sur sa marque. Il y a là des clients non satisfaits, des clients satisfaits, sans doute des collaborateurs, peut être des concurrents. Ces acteurs sont rarement identifiables. On peut distinguer les acteurs qui font une intervention de ceux qui commentent cette intervention. On observe dans le cas de l'exemple que nous avons considéré que les 49 interventions sont toutes le fait de personnes distinctes. Cela signifie que les personnes qui postent une intervention sont des acteurs qui interviennent ponctuellement. Il en va différemment des personnes qui déposent des commentaires sur les interventions postées. Les 49 messages postés ont suscité 361 réactions émanant de 218 internautes dont 156 n'interviennent qu'une fois. On peut donc observer un noyau dur d'une cinquantaine d'intervenants réguliers. L'intervenant qui intervient le plus totalise à lui seul 23 commentaires.

Il s'agit maintenant de dresser une typologie de ces intervenants. Nous avons conçu pour cela une grille d'analyse. On distingue deux dimensions selon qu'on s'intéresse à l'intervention initiale ou aux commentaires sur cette intervention. L'intervention initiale peut être positive (20\% des cas sur le corpus étudié) ou négative. Le commentaire sur une intervention peut aller dans le même sens que l'intervention originelle ou s'opposer à l'intervention de départ. Cette grille d'analyse est présentée Tableau 1 . Cette matrice permet de classer dans le cœur de la matrice (cases A, B, C, D) les internautes qui ont fait des commentaires. 
Veille d'image sur Internet...

\begin{tabular}{|l|l|l|}
\hline & $\begin{array}{l}\text { Intervention } \\
\text { initiale hostile à } \\
\text { la Caisse } \\
\text { d'Epargne }\end{array}$ & $\begin{array}{l}\text { Intervention initiale } \\
\text { favorable à la Caisse } \\
\text { d'Epargne }\end{array}$ \\
\hline $\begin{array}{l}\text { Commentaire de } \\
\text { confirmatoire } \\
\text { l'intervention initiale }\end{array}$ & A & B \\
\hline $\begin{array}{l}\text { Commentaire opposé à } \\
\text { l'intervention initiale }\end{array}$ & C & D \\
\hline
\end{tabular}

Tableau 1. Grille de profilage des intervenants

Les commentaires classés dans les cases grises correspondent à des interventions exprimées par des personnes hostiles à la Caisse d'Epargne (cases A et C), les commentaires dans les cases blanches à des personnes favorables. Les commentaires classés dans les cases $\mathrm{C}$ et $\mathrm{D}$ correspondent à des personnes introduisant une contradiction par rapport à l'avis primitivement exprimé, les commentaires dans les cellules $\mathrm{A}$ et $\mathrm{B}$ à des prises de position confirmatoires de l'idée de départ. Nous avons procédé à l'affectation manuelle de chacun des commentaires dans cette grille d'analyse.

Ce travail a été réalisé pour les 49 interventions sur le forum. Chaque personne ayant posté un commentaire apparaît donc dans le cœur de la matrice autant de fois que de commentaires postés. La matrice présentée tableau 2 permet de visualiser le poids respectif de chaque case du tableau. Les cercles sont proportionnels au nombre d'intervenants de chaque case ${ }^{4}$.

4 Nous avons choisi de rendre les résultats anonymes. En effet, bien que la source d'information soit totalement ouverte et que les acteurs interviennent par leur pseudo, ce travail d'analyse et de présentation serait de nature à révéler des profils d'acteurs. 
Dossier : Retour sur les images d'organisations

\begin{tabular}{|l|ll|l|}
\hline & \multicolumn{2}{|l|}{ Intervention hostile } & $\begin{array}{l}\text { Intervention } \\
\text { favorable }\end{array}$ \\
\hline $\begin{array}{l}\text { Commentaire } \\
\text { confirmatoire }\end{array}$ & $\mathrm{A}$ & $\mathrm{B}$ & \\
\hline $\begin{array}{l}\text { Commentaire } \\
\text { opposé }\end{array}$ & $\mathrm{C}$ & $\mathrm{B}$ & \\
\hline
\end{tabular}

Tableau 2. Grille de profilage des intervenants avec indication du nombre d'intervenant dans chaque case.

Cette matrice a été mise en œuvre de façon manuelle. Il est très difficile d'affecter ces commentaires automatiquement dans les cases de la grille pour plusieurs :

La première est que les analyses textuelles de détermination de la polarité d'un texte sont concordantes avec l'évaluation humaine dans le cas de textes longs. Dans le cas présent, les commentaires peuvent être courts. De plus, les outils automatiques ont du mal à gérer des remarques ironiques.

Enfin certaines interventions ne peuvent être classées que par l'expert. Prenons un exemple. Lorsque xavier.com dit «ça aurait été bien de préciser la ville où se trouve l'agence », il veut par cette information montrer que le mécontentement de la personne qui s'est exprimée est dû à une cause locale et qu'il ne faut pas généraliser la critique à l'entité Caisse d'Epargne. Il affaiblit donc l'argument exprimé par l'intervenant. Cette phrase, même si elle est neutre, correspond donc à une opposition au commentaire primitif.

Plusieurs points peuvent être dégagés de cette méthode d'analyse et de la mise en œuvre dont elle a fait l'objet dans l'étude de cas que nous avons considérée :

Il est rare d'obtenir des profils purs. Les acteurs qui interviennent de nombreuses fois sont rarement dans la même cellule du tableau. Néanmoins il est souvent possible d'observer une plus forte concentration d'observations pour un acteur donné dans une case particulière. Cette logique a permis d'affecter un auteur à une case et une seule du tableau.

On observe que moins d'un dixième des acteurs de la case A ont une intervention qui peut être rattachée à la case $\mathrm{D}$ et qu'il en va de même 
pour les intervenants de la case B et C. Ceci peut apparaître curieux car A comme D ont tout deux une opinion défavorable de la Caisse d'Epargne. B comme C ont de leur côté une opinion favorable.

On observe 4 intervenants (case D) exprimant une critique hostile à une prise de position favorable. A l'opposé la case C est nourrie de 19 intervenants. Elle compte de nombreux intervenants qui défendent la Caisse d'Epargne d'une intervention hostile.

Dans le cas de la Caisse d'Epargne, on observe également deux acteurs très actifs qui sont présents dans la case C. Ces acteurs sont très importants pour la Caisse d'Epargne car ils défendent particulièrement les intérêts de cette banque. On pourrait penser qu'il s'agit là de personnes payées par l'entreprise. Cela est peu vraisemblable puisqu'on retrouve des commentaires exprimés par ces mêmes intervenants dans des messages abordant des banques concurrentes 5 .

\section{Analyse}

On peut déduire de ces observations qu'il existe des forces naturelles, une logique d'autorégulation qui agit comme une «main invisible» pour contrebalancer les critiques exprimées. Sans que l'organisation ne fasse rien, des avis particulièrement négatifs vont être attaqués, les acteurs satisfaits de la Caisse d'Epargne s'investissant pour la défendre. Cette étude permet donc de voir si l'organisation a des « défenses immunitaires » suffisantes, des anticorps lui permettant de lutter contre une agression extérieure. Il est bien sûr difficile de savoir si des acteurs de la banque étudiée se cachent derrière certains intervenants. Sans doute y en a-t-il mais ceux qui interviennent le font-ils par conviction ou sont ils missionnés pour cela par leur employeur? Difficile de le savoir. Toujours est-il que la mise en place d'actions correctives spontanées, non commanditées par l'entreprise, est une hypothèse à envisager.

Au cours de l'étude du site de recommandation, nous n'avons donc pas identifié de rumeurs galopantes, le processus de transmission viral semble doté de mécanismes d'autorégulation. Les internautes agressifs voient dans bien des cas leurs actions contrebalancées par d'autres. De plus les messages déposés sur Ciao ne semblent que très peu relayés sur d'autres sites. Nous avons identifié un seul avis posté sur Ciao le

5 Xavier.com est un bon exemple d'acteur qui intervient pour atténuer les arguments d'acteurs s'exprimant contre la Caisse d'Epargne mais aussi contre des banques concurrentes. 


\section{Dossier : Retour sur les images d'organisations}

16 août 2004 et repris sur un forum de discussion le 10 octobre 2005 soit plus d'un an après. Cet exemple va à l'encontre des idées reçues sur la vitesse de diffusion d'une information sur la toile. Enfin rares sont les personnes intervenues sur Ciao qui ont déposé leur texte sur d'autres forums ou blogs. L'espace Ciao est un espace globalement étanche, une «communauté » fermée. Le web présente donc une certaine forme de compartimentation qui freine une logique de diffusion virale. Si les résultats de cette étude tendent à nuancer la portée du discours alarmiste des consultants en veille d'image sur Internet, nous ne pouvons certes pas en conclure que la surveillance de ce type de site est sans intérêt.

\section{Veille d'image dans la blogosphère}

Nous allons maintenant aborder un autre domaine du web composé des interventions dans la sphère des blogs. Nous aimerions caractériser les mécanismes par lesquels une idée nouvelle va réussir à se diffuser dans la blogosphère. On peut identifier trois mécanismes dont un spécifique aux blogs. Le premier mécanisme de diffusion est le lien hypertexte. Ce lien hypertexte permet de pointer vers une autre ressource web. La seconde caractéristique du blog est le commentaire. A la manière des forums ou des sites de recommandation, les blogs donnent la possibilité de laisser des commentaires par d'autres internautes qui deviennent acteurs. Enfin la particularité des blogs tient à la notion de rétroliens. Un rétrolien est un lien hypertexte «à l'envers » qui permet de pointer vers les ressources qui disposent d'un lien hypertexte vers vous. Le rétrolien permet donc la bidirectionnalité. Liens hypertextes, rétroliens et commentaires se conjuguent pour permettre une circulation facilitée entre des blogs, et encouragent une logique d'expression des internautes acteurs. La communication virale, si elle doit avoir lieu, trouve ici un terrain favorable.

L'étude que nous avons conduite sur les blogs a pris pour point de départ le site web Technorati.com. Ce site est un moteur de recherche de blogs qui en répertorie plus de 15 millions. Nous avons cherché à mettre en avant la communication virale de signaux faibles non détectée à partir de notre première étude. Nous allons présenter la méthodologie utilisée pour cette analyse avant de nous arrêter sur les résultats de la démarche exploratoire que nous avons conduite.

Nous avons donc interrogé le moteur http://technorati.com avec la requête « Caisse d'Epargne ». 576 résultats sont renvoyés. L'objectif était alors d'étudier chacune des interventions dans un blog, de regrouper les interventions par thème, d'étudier de quelle façon les 
interventions sont liées les unes aux autres et se diffusent dans la blogosphère.

Une grande variété de thèmes sont abordés dans les blogs. Dans le cas des blogs de la Caisse d'Epargne, les logiques de l'entreprise citoyenne, productrice, employeur sont mobilisées de façon décroissante. On est frappé de la fraîcheur de l'information. Des nouvelles arrivent chaque minute augmentant la surcharge informationnelle. Certains sujets sont ponctuels, d'autres récurrents : ils correspondent souvent à des «affaires ». Lorsque nous avons fait cette étude, les blogueurs étaient actifs sur deux sujets principaux : la généralisation du livret A aux autres banques, l'accusation d'entente illicite faite à la Caisse d'Epargne. Nous avons étudié dans le détail chacune des interventions sur ces deux sujets. Nous proposons de faire un focus sur la question de l'entente illicite. Peu importe le détail de cette affaire. Ce qui nous intéresse est la façon dont l'information primitive est relayée par la suite dans la blogosphère.

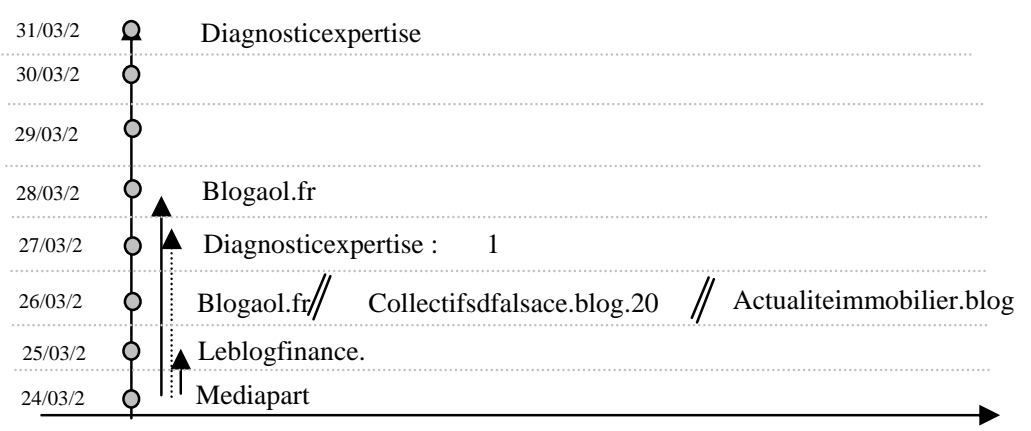

Figure 1. Visualisation des blogs actifs sur un thème particulier et de leur interaction

Le schéma de la Figure 1 reprend le nom des différents blogs qui se sont exprimés sur cette affaire On observe 8 interventions différentes sur ce thème sur 6 blogs différents. Entre la première intervention et la dernière, une semaine s'est écoulée. Deux des blogs reprenant la source de mediapart ont fait un lien hypertexte vers la page correspondante de mediapart. Un autre blog s'est contenté d'une information textuelle sans renvoi vers le site de mediapart. Il n'y a pas de rétroliens entre ces 6 blogs ni avec d'autres blogs. Il n'y a qu'un seul commentaire qui a été posté sur une de ces 8 interventions. La logique virale ne s'est donc pas déployée. Cela ne signifie pas qu'elle ne se déploie jamais. Nous avons réalisé le même type d'analyse sur d'autres le sujet de la généralisation du livret A sans observer de logique virale. 


\section{Dossier : Retour sur les images d'organisations}

A l'issue de ce travail, nous souhaitons souligner l'écart entre le potentiel d'utilisation des outils blog en particulier (et des outils web en général) et la réalité. Entre ce qui est possible techniquement et la pratique il y a sans doute une question de maturité du web 2.0 en France lorsqu' on aborde des sujets professionnels. La blogosphère n'est peut être pas suffisamment active. Nous l'avons observé dans notre validation expérimentale. Sans doute la blogosphère américaine, qui a inspiré beaucoup de nos lectures, est-elle plus active que la blogosphère française.

Il y a un autre écart entre le discours alarmiste du prestataire de service de veille et la réalité. Le web 2.0 semble comporter des mécanismes d'autorégulation qui affaiblissent la logique virale. A ce jour il est compartimenté entre des sphères qui communiquent peu. Le web n'est donc peut-être pas une jungle «sans foi ni loi », pleine de dangers prêts à s'abattre sur l'entreprise négligente. Il n'y a certes pas de charte de bonne conduite mais on croise des acteurs souvent responsables qui peuvent s'exprimer à contre-pied d'une opinion déstabilisatrice pour l'organisation pour la défendre mieux finalement qu'elle ne pourrait le faire elle-même. James Surowiecki (2008) a parlé de sagesse des foules pour caractériser ce phénomène. Cela ne veut pas dire que la question de la veille d'image sur Internet ne doit pas se poser. Elle se posera avec d'autant plus d'acuité que le web arrivera à maturité. L'acculturation à cet outil de communication n'est pas achevée et les internautes n'utilisent pour la plupart qu'une part réduite des fonctionnalités techniques offertes par l'outil.

Ce que ce travail nous a aussi montré, c'est que faire de la veille d'image sur le web oblige à se familiariser avec plusieurs communautés d'internautes. Ceci ne peut se faire qu'en s'immergeant dans leurs espaces d'échange.

Une très forte imprégnation humaine du sujet doit être associée à la logique automatique. Le veilleur devra faire appel à son expertise ou à son intuition pour pallier les insuffisances techniques de collecte des informations et juger des résultats signifiants pour l'entreprise. Un compte rendu automatique renvoyé régulièrement a peu de chance de fournir à l'organisation une vision éclairée des risques encourus et des moyens d'y faire face. 
Veille d'image sur Internet...

\section{Bibliographie}

BARNETT M.L., "Corporate reputation : the definitional landscape", in Corporate Reputation Review, vol.9, 2006, pp. 26-38

CIPOLLONE P., Boiled Frogs and Your Organization's Reputation, white paper, société Factiva, 2006, http://www.informationmanagement.com/specialreports/20051011/1038938-1.html consulté le 15 avril 2009.

CHRISTIE D.J., "A trilateral model for management of corporate image: an examination of the interrelationship between an organization's Self image, its projected image and its perceived image", Thèse de doctorat, Université Griffith, 2002, 324 pages.

FESTINGER L., A Theory of Cognitive Dissonance. Stanford, CA : Stanford University Press, 1957, 308 pages.

General Inquier, (2000)

http://www.wjh.harvard.edu/ inquirer/3JMoreInfo.html

HEUER R. J., "Psychology of Intelligence Analysis" History Staff Center for the Study of Intelligence Central Intelligence Agency, 1999, 214 pages.

JODELET D., Les représentations sociales, Paris : PUF, 1989, 429 pages.

JURVETSON, S. AND DRAPER, T., Viral Marketing phenomenon explained, Draper Fisher Jurvetson, 2000,

http://www.dfj.com/news/article 26.shtml consulté le 15 avril 2009

KAHNEMAN D., SLOVIC P. ET TVERSKY A., Judgement Under Uncertainty: Heuristics and Biases, Cambridge University Press, 1982, pp.1124-1131.

KAY, J., The Truth about Markets, London: Penguin, 2004, 496 pages.

LACEY A., "A Simple Probabilistic Approach to Ranking Documents by Sentiment", Proceedings of the Conference Class of 2005 Senior Conference on Natural Language Processing, Spring 2005 Swarthmore College, 2005, pp.1-7.

MOSCOVICI S., «Psychologie des représentations sociales», in Cahiers Vilfredo Pareto (14), Paris, 1976, pp. 409-416.

SUROWIECKI J., La sagesse des foules, Paris : Editions Jean-Claude, 2008, 384 pages 
Dossier : Retour sur les images d'organisations

Swarthmore, Pennsylvania, USA,

http://www.cs.swarthmore.edu/ richardw/cs97/papers/01_Paper.pdf

MEVEL O., Du rôle des signaux faibles sur la reconfiguration des processus de la chaîne de valeur de l'organisation, Thèse de doctorat en sciences de gestion, 2004, 459 pages.

ROBSON D., «Cognitive Rigidity: methods to overcome it », Actes $d u$ colloque 2005 International Conference on Intelligence Analysis, 2-4 mai 2005, https://analysis.mitre.org/proceedings/Final_Papers_Files/40_Camera Ready_Paper.pdf consulté le 15 Avril 2009

TVERSKY A. ET KAHNEMAN D. (1974), «Judgment Under Uncertainty: Heuristics and Biases », Science, 185, 1974, pp. 11241131.

WILSON, R.F., "The Six Simple Principles of Viral Marketing, Web Marketing Today, from http://www.wilsonweb.com/wmt5/viralprinciples.htm , 2000.

WHISSELL, C. M., "The dictionary of affect and language", In Plutchik, R. and Kellerman, H., editors, Emotion: Theory, Research, and Experience, volume 4: The measurement of emotions, Academic Press, New-York, 1989, pp. 113-131 